\title{
Internet Lite for Sustainable Development
}

\author{
Josef Noll ${ }^{1,2}$, Wisam A. Mansour ${ }^{1, *}$, Christine Holst $^{3}$, Sudhir Dixit ${ }^{1}$, \\ Felix K. Sukums ${ }^{4,9}$, Helena A. Ngowi ${ }^{5}$, Danica Radovanovic ${ }^{1}$, \\ Elibariki R. Mwakapeje ${ }^{6}$, Maurice Isabwe ${ }^{7}$, Andrea S. Winkler ${ }^{3,8}$ \\ and Bernard Ngowi ${ }^{4}$
}

${ }^{1}$ Basic Internet Foundation, Kjeller, Norway

${ }^{2}$ Dept. of Technology Systems, University of Oslo, Oslo, Norway

${ }^{3}$ Centre for Global Health, Institute of Health and Society, University of Oslo, Oslo, Norway

${ }^{4}$ Muhimbili Medical Research Centre, National Institute for Medical Research (NIMR), Dar es Salaam, Tanzania

${ }^{5}$ Dept. of Veterinary Medicine and Public Health, Sokoine University of Agriculture, Morogoro, Tanzania

${ }^{6}$ Dept. of Food Safety and Infectious Biology, Norwegian University of Life Sciences (NMBU), Adamstua Campus, Oslo, Norway

${ }^{7}$ Dept. of Information and Communication Technology, University of Agder, Grimstad, Norway

${ }^{8}$ Center for Global Health, Department of Neurology, Technical University of Munich, Germany

${ }^{9}$ Muhimbili University of Health and Allied Sciences, Dar es Salaam, Tanzania

* Corresponding Author

Received 09 December 2018;

Accepted 10 December 2018

\section{Abstract}

The Digital Divide is one of the key challenges for mankind. Despite attempts to digitally include people, the divide is bigger than ever. This contribution [1] to a sustainable future promotes "Internet lite" as the new paradigm for providing everyone with the opportunity to participate in the digital society. The current paper first addresses the reasons for the digital divide, thereafter addresses free access to information as catalyst for the sustainable development goals (SDGs) and provides the path towards "Internet lite for all".

Journal of NBICT, Vol. 1, 223-238.

doi: 10.13052/nbjict1902-097X.2018.012

This is an Open Access publication. (c) 2018 the Author(s). All rights reserved. 
Keywords: Digital inclusion, sustainable development, information spot, digital literacy, Internet lite, and Internet.

\section{Introduction and Background}

The Internet has developed from information distribution to become the business enabler, indicated by the fact that the top 6 largest companies by market value are all IT-companies [2]. However, the gap between developed and developing countries is still wide. Access to Internet is a commodity in Europe, while only a minority of people in Africa have the capability to participate in the digital society.

According to ITU [3], Nearly $90 \%$ of young individuals are not using the Internet in Africa, Asia and the Pacific. Giving the move to digital industries, access to information is not a question of affordability, it is a question of sustainability.

As ITU further points out, $84.2 \%$ of households in Europe have Internet access, while only $18 \%$ of households in Africa have Internet access. In developed countries, 94\% of young people aged 15-24 use the Internet, as compared to only $30 \%$ in least developed countries (LDCs). This digital gap, leaving $70 \%$ of young people in LDCs without an opportunity to participate in the digital life, is one of the reasons for migration from rural to urban areas, or even further.

\section{Internet Lite for All}

Our understanding is that digital inclusion should address everyone in the society, and that everybody shall have free access to information. "Internet

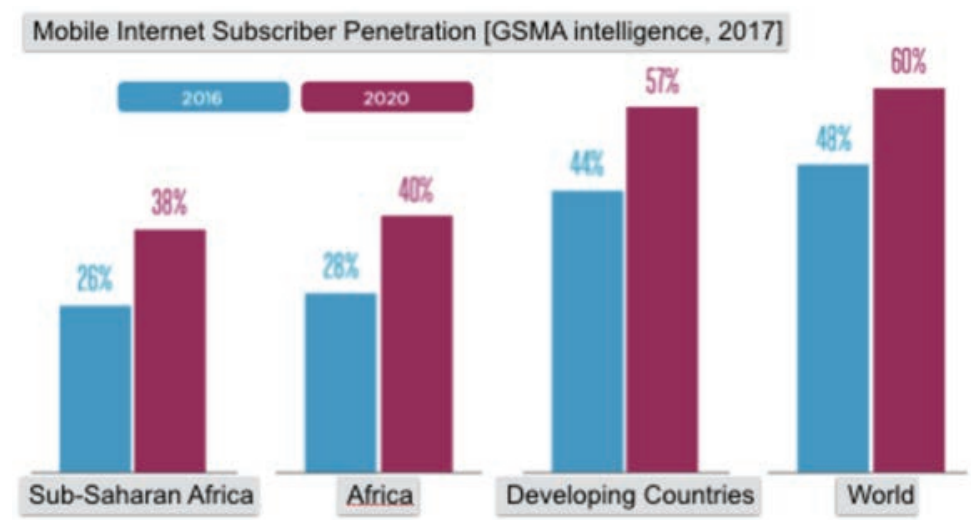

Figure 1 Mobile Internet Penetration from 2016 to 2020. 


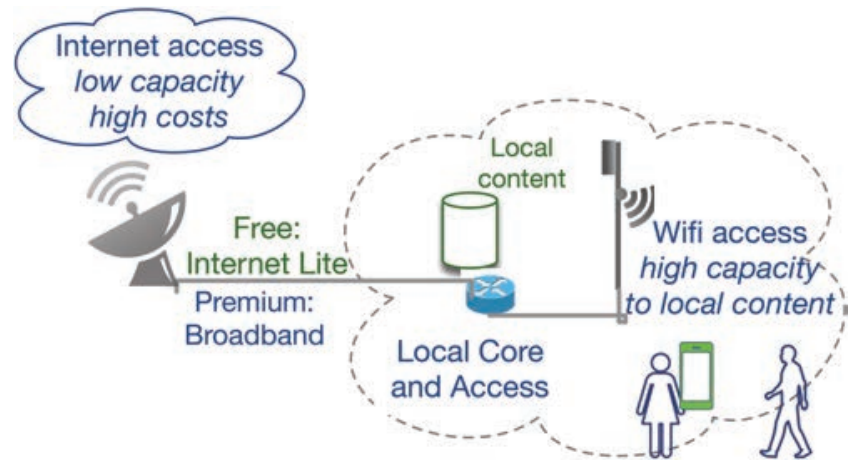

Figure 2 Freemium concept for Internet Lite for All.

access is a human right", and free access to information, named "Internet lite for all", is the way to establish a sustainable solution. The basic model is adapted from commuting on roads: pedestrians and cyclists can use the road for free, while car and truck drivers have to pay. This freemium (free \& premium) model can be applied for Internet access, with free access to information, and premium access to entertainment, games and other broadband content.

From our experiences in project activities, especially the "Nondiscriminating Access for Digital Inclusion" (DigI) project [4], we see that an "Information Spot" per village is the core concept for digital inclusion and societal empowerment. Our developments suggest that the information spot should contain a village/society server carrying information and providing free access in the society. An example of such a village server is developed during the DigI project, being available at yeboo.com. Figure 2 provides a sketch of the Local Information Spot, here with an integrated Wifi hot-spot connected to the local core network.

\section{Traditional Business Models are not Sufficient to Include All}

Though Mobile Operators see a continuous rise the number of SIM cards, with a raise of unique Mobile Subscribers from 43\% (2016) to 50\% (2020), the expectations from the Groupe Spéciale Mobile Association (GSMA) on the Mobile Economy [5] shows that the mobile internet subscriber penetration in Sub-Saharan Africa (SSA) will reach only about 38\% in 2020. This means that about $2 / 3$ of the population in SSA will not be part of the digital economy.

There are several reasons why digital inclusion lacks uptake [6] in SSA: (i) phone prices, (ii) availability and affordability of the mobile broadband 
network coverage, (iii) traffic speed and (iv) lack of local content. While (i) phone prices fall continuously, with smartphones being available in the 20-30 US\$ price range, (ii) availability of the mobile broadband network coverage leaves out behind. Operators have only limited funds for network deployment, and thus mobile broadband networks will be first deployed where there is the highest return on investment. The respective governments focus often on voice connectivity $(2 \mathrm{G})$, thus leaving mobile broadband network deployment as an answer to sufficient return of investment instead of ROI.

Second, customers with as little as 1-2 US\$/month for communication purposes are of little interested for mobile operators, as the cost of customer registration into the operators infrastructure exceeds 60 US\$. Thus, operators can't profit from people with ultra-low income, and rather focus on people higher up in the pyramid. Third, operators focus on content which is attractive to people, i.e. entertainment, sports, and games.

Fourth, access to non-profit information such as health and education will loose when competing with commercial content.

As a result, almost half of the mobile connections (44\%) will be $2 \mathrm{G}$ connections by 2020 in Eastern Africa (EAC), according to GSMA [5]. Given the low capacity networks, and related them to the lack of local content ( $i v$ ) and lack of (digital) literacy are other factors hampering the uptake of mobile broadband.

An information spot in each village, combined with a village server, will address topics (ii) to (iv) directly, and through fixed installed tablets enable the access to the Internet for $(i)$ people without smartphones. Our example of established information spot at kjeller (see Figure 3) has been rolled-out in 3 villages in Tanzania, carries digital health information [7], alongside with information from the village leaders and social network content provided by users themselves.

As the health information is supported by animation and videos and presented using Tanzanian Swahili, it contributes to digital literacy. Though, our vision for a sustainable future is that NGOs, Universities, companies and societies will contribute with contextualised content reflecting the needs of communities.

\section{Internet Lite as a Catalyst for the SDGs}

The SDGs and especially Targets 9.C "Significantly increase access to information and communications technology, and strive to provide universal and affordable access to the Internet in least developed countries by 2020" and 


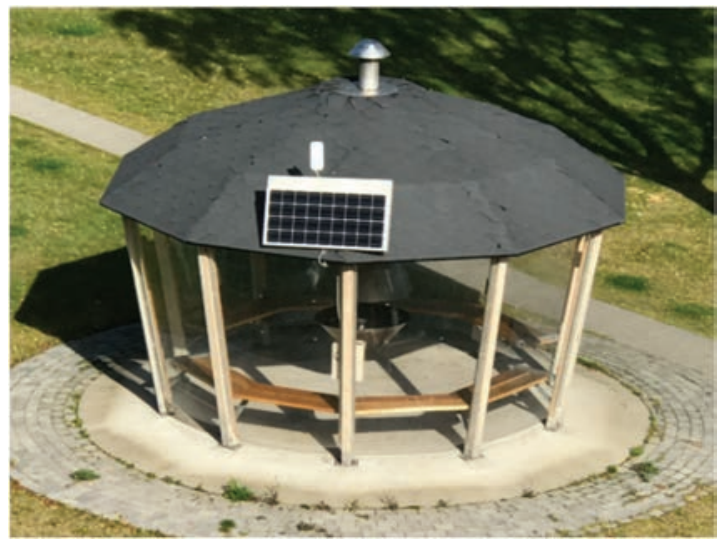

Figure 3 Prototype of health spot at Kjeller, Norway.

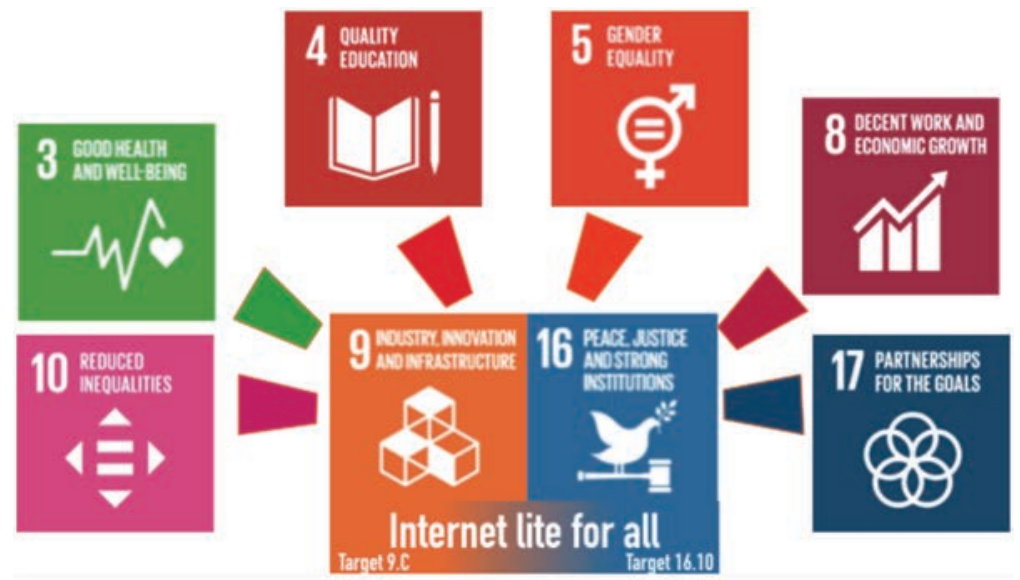

Figure 4 "Internet Lite", addressing Target 9.C and Target 16.10, as catalyst for the SDGs.

Target 16.10 "Ensure public access to information and protect fundamental freedoms..." are the core drivers for our work. Information spots with "Internet lite for all" will directly answer Target 16.10, and enable the access to information and communications in areas where it is not economically viable to deploy mobile broadband.

The next chapter will bring an example of how digital health information will contribute to SDG 3 "Good Health and Well-Being" as an example why "Internet lite for all" could be the catalyst for the majority of the SDGs. We would especially emphasise digital information for education in SDG 4, empower women and girls through information (SDG 5), digital jobs and 
participation in the digital economy (SDG 8), as well as strong contributions to SDG 10 on reduced inequalities with and amongst countries. We envision everyone to contribute with information on the village platform, and thus contribute to the digital partnership in SDG 17.

\section{Digital Health for Health Knowledge and Digital Literacy}

The spread of digital technologies and global interconnectedness has a significant potential to accelerate progress towards achieving the health-related SDG 3 at a global level. The primary focus has been on health information systems, giving a clear benefit for governments and societies. However, health information systems have a tendency to neglect people and societies that are digitally excluded.

It has clearly been recognised that digital divides influence digital literacy, engagement in social and educational life, and therefore overall participation and collaboration in an online society $[8,9]$. Our approach is to use digital health information to promote early access of health care services and the application of preventative strategies, ultimately reducing morbidity and mortality.

The village platform residing at our information spot carries specific health information [10], being freely available to everyone. We are currently performing an intervention study in the villages of Izazi and Migoli in the Iringa district in Tanzania to develop health messages, promote the messages and assess the effect of digital health promotion. The selected health messages address HIV/AIDS, tuberculosis (TB) and Taenia solium cysticercosis/taeniosis (TSCT).

Ongoing work evaluates key performance indicators (KPIs) for assessing both digital literacy and health knowledge uptake in order to evaluate digital health promotion as compared to traditional paper-based health information.

\section{Technical Realisation of Internet Lite}

As stated above, the network is often the limiting factor, being low-capacity $2 \mathrm{G}$, congested $3 \mathrm{G}$ or a satellite link limited traffic (see Figure 2). Providing "internet lite for all" in an "Information Spot" is our contribution to the SDGs. Following the principles of net-neutrality, we suggest filtering after content type, with information (text, pictures and local content) being free, while broadband content such as entertainment, video and games are premium 
content. Providing a local core network as shown in Figure 5 will enable the functionality of Internet Lite. Filtering of content types is happening in the local core network (see Figure 6), which also provides the village server. The concept is relatively inexpensive, our realisation costs around 300 US\$ for the local core network, including solar panel and battery for operation and charging of devices.

Filtering between free and premium content is performed at the local network control centre (LNCC), which also supports the village server. Filtering information for the LNCC is provided through the core network infrastructure, provided by the Basic Internet Foundation. The core network infrastructure supports also voucher generation for the premium access to information. In our cost analysis [6], we pointed out that Internet Lite can serve up to 300 users over a $1 \mathrm{Mbit} / \mathrm{s}$ line, with a cost of 7 US\$ per month for visiting around $100 \mathrm{Web}$ pages ${ }^{1}$. Using traditional Internet access, only 50 users could be served, with costs of 38 US $\$$ for visiting 100 Web pages.

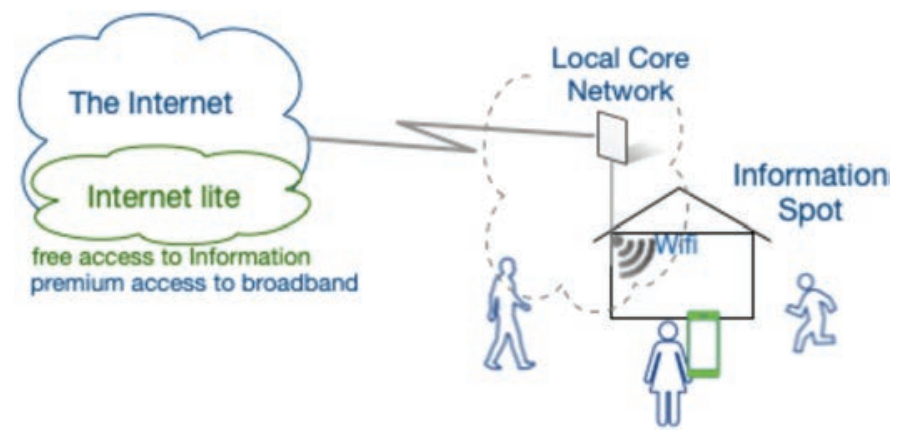

Figure 5 Village information spot with local core network.

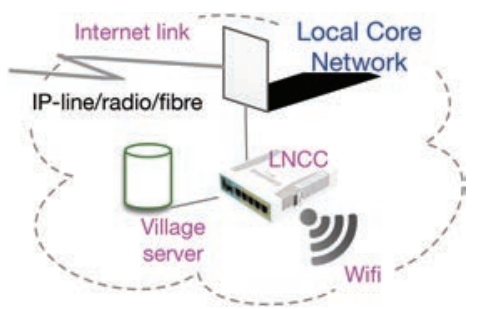

Figure 6 Local Core Network with village server and local network control centre (LNCC).

\footnotetext{
${ }^{1}$ The cost analysis [6] is performed using a $1 \mathrm{Mbit} / \mathrm{s}$ satellite link costing $2000 \mathrm{US} \$ / \mathrm{month}$ as an example.
} 


\subsection{Call for Collaboration and Conclusions}

Our multi-cultural and multi-dimensional team with 11 partners from 8 countries in the DigI project has established digital health information for (i) contributing to better health, (ii) supporting digital literacy, and (iii) demonstrating digital information access as catalyst for the SDG 3.

We see the importance of free access to information, addressing directly the Targets 9.C and 16.10 of the SDGs, as the catalysts for the sustainable development goals. Our concept of one information spot per village provides free access to information, following the vision "Internet lite for all". In order to ensure net-neutrality, our approach of Internet lite provides content type filtering, with free access to text, pictures and local video, and premium access to video, voice, games and other entertainment content.

The health spots for Africa have costs of roughly $\$ 300$. Even on a $1 \mathrm{Mbit} / \mathrm{s}$ line we can provide 300 people with free access to information, using either locally available tablets or their own smart-phones, and in addition light and power to charge the phones.

Given the societal advances in digital literacy, digital inclusion and the participation in the digital society, Internet lite is seen as the enabler to connect the unconnected 3.5 billion people on the planet and to become a robust catalyst for the SDGs. We envision everyone to contribute with information on the village platform, and thus add to the digital partnership in SDG 17.

\section{References}

[1] This work was supported by the Research Council of Norway through the Visjon2030-mechanism, project "Non-discriminating Access for Digital Inclusion", project number 267558.

[2] The 100 largest companies in the world by market value in 2018, https://www.statista.com/statistics/263264/top-companies-in-theworld-by-market-value/

[3] ITU, ICT Facts and Figures 2017, https://www.itu.int/en/ITU-D/ Statistics/Documents/facts/ICTFactsFigures2017.pdf

[4] The "Non-discriminating Access for Digital Inclusion" project: http://DigI.BasicInternet.no

[5] GSMA, The Mobile Economy, Sub-Saharan Africa 2017, https://www. gsmaintelligence.com/research/?file=7bf3592e6d750144e58d9dcfac6ad fab\&download 
[6] Noll, J., Dixit, S., Radovanovic, D., Morshedi, M., Holst, C., and Winkler, A. S. (2018). 5G network slicing for digital inclusion. In IEEE 10th International Conference on Communication Systems \& Networks (COMSNETS) pp. 191-197.

[7] Village platform with dedicated health information https://yeboo.com/ health_information_dashboard.php

[8] Van Deursen, A., and Van Dijk, J. (2011). Internet skills and the digital divide, New Media \& Society, 13(6), pp. 893-911. [Online]. Available: https://doi.org/10.1177/1461444810386774

[9] Health Information, as provided through the DigI project in Tanzania: https://yeboo.com/health_information_dashboard.php

[10] Radovanovic, D., Hogan, B., and Lalic, D. (2015). Overcoming digital divides in higher education: Digital literacy beyond facebook, New Media \& Society, 17(10), 1733-1749. https://doi.org/10.1177/146144481 5588323

\section{Biographies}

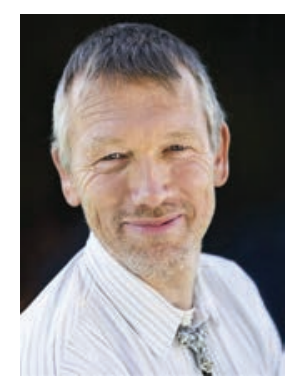

Josef Noll is professor at the University of Oslo (UiO) and Secretary General of the Basic Internet Foundation. His focus is on communication and IoT-/Information security, and especially digitalisation based on free access to information for all. His vision of "Internet Lite for all" addresses directly the Sustainable Development Goals (SDGs) 9.C and 16.10 of the Agenda 2030. His focus is on how Internet Lite catalysts a sustainable development, especially in the areas health (SDG 3), Education (SDG 4), empowerment (SDG 5) and decent work (SDG 8). 


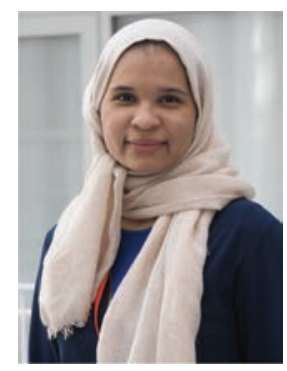

Wisam A. Mansour is a digital entrepreneurship advisor at the Basic Internet Foundation. She hold a master degree from the University of Oslo, Universitetet i Oslo, in Innovation and Entrepreneurship, 2018. For her master thesis, she did an indeep study on facilitating women entrepreneurship in the Global South. Her field of research focusing on entrepreneurship in developing economies with special focus on women entrepreneurship empowering. She is working on developing business courses and entrepreneurial platform for developing countries (EntrepNet).

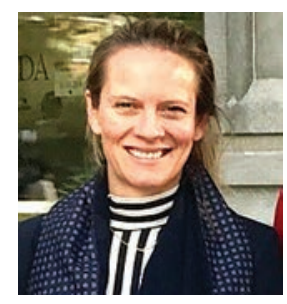

Christine Holst received a bachelor of business administration from BI Norwegian Business School, Oslo, Norway; thereafter a B.Sc in African studies and MPhil in development studies, specializing in Geography from Norwegian University of Science and Technology, Trondheim, Norway. Today she holds the position as a Doctoral research fellow, at the Centre for Global Health, Institute of Health and Society, University of Oslo, Norway. She is the coordinator of the Non-Discriminating access for digital inclusion (DigI) project. 


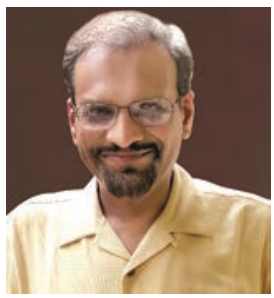

Sudhir Dixit is a Senior Fellow and Evangelist of Basic Internet at the Basic Internet Foundation in Norway and heads its San Francisco office. From December 2009 to 2015, he was a Distinguished Chief Technologist and CTO of the Communications and Media Services for the Americas Region of Hewlett-Packard Enterprise Services in Palo Alto, CA, and prior to this he was the Director of Hewlett-Packard Labs India. Before joining HP, he worked with BlackBerry as Senior Director, with Nokia and NSN as Senior Research Manager, Nokia Research Fellow, Head of Nokia Research Centre (Boston), and Head of Network Technology (USA), and Verizon as Staff Director and Principal Scientist. Dixit has a PhD from the University of Strathclyde and MBA from the Florida Institute of Technology, He is a Fellow of IEEE, IET and IETE and a Distinguished Lecturer at the IEEE Communications Society.

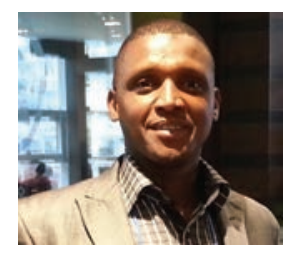

Felix K. Sukums is a Lecturer, Researcher, Senior Consultant and Director of Information and Communication Technology (ICT) at the Muhimbili University of Health and Allied Sciences (MUHAS) in Dar es Salaam, Tanzania. He holds a PhD in Medical Informatics from Heidelberg University, Germany since April 2016. He also holds Master of Science and Bachelor of Science degrees in Computer Science from the University of Dar es Salaam in Tanzania awarded in 2009 and 2002 respectively.

Sukums has led numerous information systems, digital health research and consultancies projects/assignment including the Tanzania National eHealth Strategy, electronic population registration system, human resource information system, use of clinical decision support systems for improving maternal 


\section{Josef Noll et al.}

and new-born care and telemedicine. He coordinates UNESCO Chair on Telemedicine at MUHAS.

$\mathrm{He}$ is currently working with the Muhimbili Medical Research Centre, National Institute for Medical Research (NIMR), Dar es Salaam, Tanzania in the design and implementation of the Digital Inclusion (DigI) project aming to deploy "Internet Lite for All".

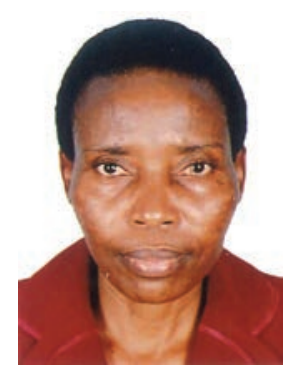

Helena A. Ngowi from Sokoine University of Agriculture, Tanzania, is a specialist in veterinary-public health and a consultant in the health promotion approach to the control of parasitic diseases transmissible between animals and humans. She has conducted several high profile research studies on the prevalence, transmission and risk factors of porcine cysticercosis caused by Taenia solium. Recently, she has explored whether school children can transmit health messages of $T$. solium effectively to the community, whereby she has employed a combination of qualitative and quantitative research methods through a social science approach. H Ngowi has also trained in epidemiological and qualitative research and has developed different health education materials including leaflets, posters, training manuals and video programmes. Currently, H Ngowi is co-supervising a research to assess the impact of digital health information access on the control of $T$. solium infections in pigs in selected rural villages of Tanzania, the study funded by Norwegian Research Council through DIGI project. 


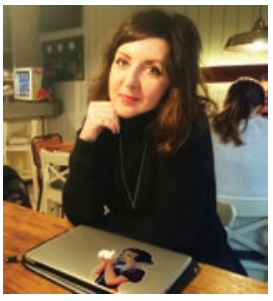

Danica Radovanović is a digital inclusion specialist and senior researcher at the Basic Internet Foundation, Norway. She is a PhD Chevening Scholar, Oxford Internet Institute, University of Oxford, and a doctorate graduate from the Faculty of Technical Sciences, Novi Sad. Radovanović worked on international Internet and Web projects at the United Nations (FAO), Library of Congress (DC) European division, was involved in a global citizen initiatives Global Voices and Rising Voices, and other digital cooperation projects. She is a project manager at the ISOC (Internet society) project DUGG on gender digital divide. Her work focuses on deploying digital inclusion and digital literacy skills programs, KPI for digital inclusion, digital literacy initiatives, and internet infrastructures to the unconnected areas. Radovanovic is the recipient of the Best academic work - dissertation award in 2015. in Serbia. She is an ISOC member and DITA IEEE member.

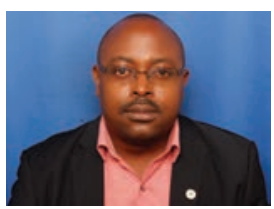

Elibariki R. Mwakapeje, has pursued a Bachelor degree (Hons) in Environmental Health Sciences from the University of Dar es Salaam (2004), Masters degree in Applied Epidemiology from the Muhimbili University of Health and Allied Sciences (MUHAS) in 2010 and currently, he is a Ph.D researcher at the Centre for Epidemiology and Biostatistics, Department of Food Safety and Infection Biology of the Norwegian University of Life Sciences in Oslo, Norway since April 2016. His Ph.D research topic is entitled, "Use of a One Health Approach for understanding the Epidemiology and Management of anthrax outbreaks in the humans-livestock-wildlife and environmental health interface areas of Northern Tanzania" - Scheduled for a Public defense on 22nd February 2019. 


\section{Josef Noll et al.}

Elibariki Reuben Mwakapeje is a Senior Epidemiologist and has worked for the Ministry of Health, Community Development, Gender, Elderly and Children in Dar es Salaam, Tanzania since 2005. Initially, he worked as a Diseases Surveillance Officer involving himself in investigating diseases outbreaks, diseases surveillance, coordinating rapid response teams (RRT) at national, regional and district levels during response to disease outbreaks and other public health emergencies of national and international concerns.

Later on, he was appointed as a National Coordinator for the Integrated Diseases Surveillance and Response (IDSR) in 2010 at the same Ministry, with this position he was responsible for ensuring that both indicator and event based surveillance systems are well implemented and streamlined as per the International Health Regulation (IHR 2005).

Moreover, in 2011 he participated in the establishment of the diseases surveillance systems in the cross border districts within the East African Community region in order to ensure for a well-structured and resilient systems for prevention, detection and response to various public health threats in the region. Most importantly, in 2013, Elibariki was one of the founders for the electronic diseases surveillance systems (eIDSR) in Tanzania. This system was established in order to foster for a robust real - time surveillance systems as a requirement for both Global health Security Agenda (GHSA) and International Health Regulation (IHR 2005) in the country.

Elibariki is currently working on to establish a project for ensuring free access to digital health information through smartphone application in Northern Tanzania. This project intends to provide digital health information as a userfriendly tool for behavior change leading to effective prevention and control of anthrax outbreaks in the marginalized Maasai communities in Monduli district of northern Tanzania.

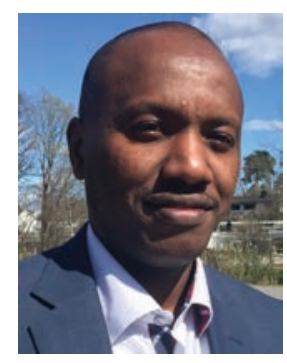

Maurice Isabwe is an Associate Professor of Multimedia \& eLearning at the Faculty of Engineering and Science, University of Agder, Norway. He is a founding CEO of Future Competence International Ltd, a forward-thinking 
futuristic enterprise thriving to maximize the human capital through competence development services \& products. Isabwe contributes to the development of formal and non-formal digital learning solutions addressing global digital inclusion and lifelong learning. He consults on ICT development projects and contributes in systems design with his expert competence in the field of Human-Computer Interaction. He holds a $\mathrm{PhD}$ in Information and Communication Technology from the University of Agder, specialising in human-centred design of interactive systems and mobile learning. His academic interests include Interaction Design; Open, Distance and e-Learning; Visualisations \& Simulations in mathematics education and Gamification. For the last 16 years, he has been working with universities in East Africa, the United Kingdom and Norway.

Isabwe's research focuses on usability and user experience for the web, mobile, wearables, augmented and virtual reality technology. His work involves post-graduate teaching and supervision of research projects in the area of Human Computer Interaction and Technology Enhanced Teaching and Learning. He has contributed to the establishment of a Master program in Multimedia \& Educational Technology at University of Agder and a blended learning master program in Instructional Design and Technology at Makerere University (Uganda).

He is a reviewer of scientific journals, convener and a regular speaker at international conferences in the field of interaction design and digital technology in higher education. He has published more than 60 scientific articles contributing to the advancement of online education, blended learning and human-centred digital solutions.

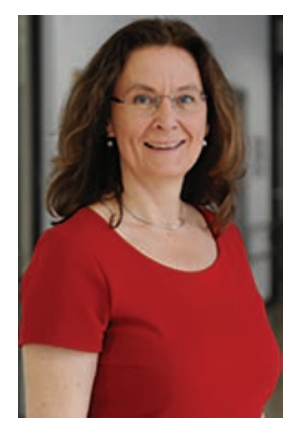

Andrea S. Winkler, $\mathrm{MD}, \mathrm{PhD}$, is a specialist neurologist and the director of the Centre for Global Health at the University of Oslo, where she also holds a full professorship in Global Health. She is also the co-director of 
the Center for Global Health at the Technical University of Munich. Prof. Winkler has over 15 years of experience with both clinical work and research in countries of sub-Saharan Africa. Her special interest lies with povertyrelated neglected diseases of the infectious as well as non-infectious nature, global neurology/mental health and global digital health. She had/has leading roles in various large-scale multidisciplinary health consortia in sub-Saharan Africa funded by the German Research Foundation, the Bill and Melinda Gates Foundation, the Norwegian Research Council and the Germany Ministry of Education and Research, among others.

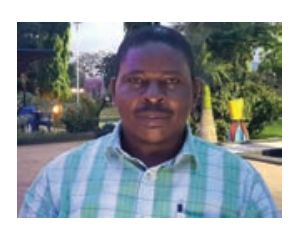

Bernard Ngowi is epidemiologist and a public health specialist with 14 years experience in HIV/AIDS, Cysticercosis, opportunistic infections and tuberculosis research. Ngowi has five years' experience working as a physician in remote rural Tanzania, having conducted epidemiological studies in HIV/AIDS, Cysticercosis, Tuberculosis and Opportunistic infections in Tanzania. Ngowi is a Principal Researcher at Tanzania National Institute for Medical Research Muhimbili Centre. Currently Ngowi is conducting HIV and cysticercosis related studies in Tanzania which includes the evaluation of an antibody detecting point-of-care test for the diagnosis of Taenia solium taeniasis and (neuro) cysticercosis in communities and primary care settings of highly endemic, resource-poor areas in Tanzania and Zambia. Ngowi is a Principal investigator for the Cysticercosis Network of Sub-Saharan Africa; CYSTINET Africa. B Ngowi is a member of the East African Consortium for Clinical Research and a monitoring and evaluation officer of the Afrique One ASPIRE Consortium. 